\title{
ON THE DENSITY OF COLOR-FAMILIES
}

\author{
E. We $\backslash z 1$ \\ Institut für Informationsverarbeitung \\ Technische Universität Graz \\ Steyrergasse 17 \\ A-8010 Graz/Austria
}

\section{Introduction}

Recent research of Maurer, Salomaa, and Wood, [2], in the area of finite grammar forms has established a connection between form theory and the theory of graphs.

More specially, the mechanism of interpretation - applied to languages containing only words of length two - comes out to be a generalization of the notion of $n$-coloring if the languages at issue are interpreted as graphs in the natural way (letters become vertices, a word 'ab' represents an edge from a to b). Thus, considerations concerning graphs yield results which can be applied to finite grammar forms, afterwards. In this paper we present an outline of the proof that color-families are dense (a color-family consists of the interpretations of an undirected graph). By virtue of the above mentioned connection of graph theory and form theory this result can be used to establish the density of certain families of finite grammar forms (see [4]).

For unexplained notions in graph theory I refer to [1]. [6] is a genera] introduction to the theory of forms.

\section{Preliminaries}

We consider finite, undirected, unlabeled graphs, possibly with loops, but without parallel edges.

An elementary homomorphism in such a graph $G$ consists of identifying two vertices $x$ and $y$ and inserting edges between the identified vertex $x=y$ and $a 11$ vertices $z$ adjacent to ejther $x$ or $y$ in $G$ (when $x$ and $y$ are adjacent, $x=y$ has a loop - this means an edge from the vertex to itself). A graph G' is a morphic image of a graph $G$ if it is obtained from $G$ by finitely many elementary homomorphisms.

Note that this elementary homomorphism, finitely often applied, is an inverse dfl-substitution ( $\mu$ is a disjoint-finite-zetter substitution on 
an alphabet $v$, if $\mu(v)$ is a finite set of letters for all $v$ in $v$ and $v \neq w$ implies $\mu(v) \cap \mu(w)=\emptyset)$. Therefore the following definition is natura 1:

A graph $G$ is an interpretation of a graph $H$ if a morphic image $G$ ' of $G$ is a subgraph of $H$.

A color-famizy $\mathscr{L}(G)$, defined by a graph $G$, is the set of al1 graphs $H$ which are interpretations of $G$.

obviously, the relation $\mathcal{L}(G) \subseteq \mathcal{L}(H)$ holds if and only if $G$ is an interpretation of $H$, since 'interpretation' is transitive. Moreover, the relation 'interpretation' is decidable. Let $k_{n}$ be the complete graph with $n$ vertices and let $c_{m}$ be the cycle with m vertices. Then we have a basic hierarchy among color-families established in [2]:

$$
\begin{aligned}
\mathscr{L}\left(k_{2}\right) \subsetneq & \ldots \mathscr{L}\left(c_{2 m+1}\right) \subsetneq \mathcal{L}\left(c_{2 m-1}\right) \subsetneq \ldots \mathcal{L}\left(c_{3}\right)=\mathcal{L}\left(k_{3}\right) \subsetneq \\
& \subsetneq \mathcal{L}\left(k_{4}\right) \subsetneq \ldots \mathcal{L}\left(k_{n}\right) \subsetneq \mathcal{L}\left(k_{n+1}\right) \subsetneq \ldots
\end{aligned}
$$

Concerning this hierarchy the question arises (especially from the point of view of grammar forms) whether there are color-families strictiy between these $c_{2 m+1}$ 's and $k_{n}{ }^{\prime} s$, or more general, whether for every pair of families

$\mathcal{L}(G) \subsetneq \mathcal{L}(H)$

there is a family $\mathscr{L}(F)$ for which

$$
\mathcal{L}(G) \subsetneq \mathcal{L}(F)_{\subsetneq} \mathcal{L}(H)
$$

holds, i.e. whether color-families are dense. If such a family $\mathcal{L}(F)$ does not exist, we say $G$ is a predecessor of $H$.

\section{Outline of the Proof of the Density of Color-families}

MAIN THEOREM: Color-families are dense (excluding the pair $\mathcal{L}\left(K_{1}\right), \mathcal{L}\left(K_{2}\right)$ ). Lemma 1:

(i) If $P$ and $Q$ are predecessors of a connected graph $G$, then

$$
\mathcal{L}(P)=\mathcal{L}(Q) \quad \text { holds. }
$$

(ii) Let $H$ be a graph with $\mathcal{L}(H) \subset \mathcal{L}(G)$, and let $P$ be a predecessor of $G$. Then $H$ is an interpretation of $P$.

Proof(outline): For two predecessors $P$ and $Q$ the relations $\mathcal{L}(P) \subseteq \mathscr{L}(0)$ or $\mathscr{L}(P) \neq \mathcal{L}(Q)$ would be a contradiction to the definition. But if $\mathscr{L}(P)$ and $\mathscr{L}(Q)$ are incomparable, we have a contradiction with the relation $\mathscr{L}(P) \subsetneq \mathcal{L}(P \cup Q) \subsetneq \mathcal{L}(G)$, too $(P \cup Q$ is the disjoint union of the graphs $P$ and $Q$ ). Consequently, $\mathcal{L}(P)$ equals $\mathcal{L}(Q)$. Proof of (ii) is similar. a 
If $\mathscr{L}(W)$ is a subset of $\mathscr{L}(G)$ and the relation $\mathcal{L}\left(W^{\prime}\right.$ If $\mathcal{L}(G)$ holds for no morphic image $W^{\prime}(\neq W)$ of $W$, then $W$ is called a weak predecessor of $G$. The following theorem shows the interrelation between predecessors and weak predecessors.

Theorem 2:

Let $G$ be a connected graph. G has no predecessor if and only if $G$ has infinitely many weak predecessors (Two weak predecessors $V$ and $W$ are termed different if $\mathscr{L}(V) \neq \mathcal{L}(W)$.

Proof: $(\leftarrow)$ Let $W$ be a weak predecessor of $G$ and let $P$ be a predecessor of $G$. Then we know (see Lemma 1 ) that $W$ is an interpretation of $P$. Thus there is a morphic image $W^{\prime}$ (of $W$ ) which is a subgraph of $P$. Then

$$
\mathscr{L}\left(W^{\prime}\right) \subseteq \mathscr{L}(P) \subset \mathcal{L}(G)
$$

contradicts the definition of 'weak predecessor', except $W$ ' equals $W$. Therefore a 11 weak predecessors of G are subgraphs of the predecessor $P$. obviously, no finite graph $P$ can have infinitely many weak predecessors as subgraphs.

$\Leftrightarrow$ Let $W_{1}, W_{2}, \ldots W_{n}$ be a 71 the weak predecessors of $G$. It is easily seen that a graph $H$ with $\mathcal{L}(H) \subsetneq \mathcal{L}(G)$ has a morphic image $H^{\prime}$ which is a weak predecessor of $G$. Consequentiy such a graph $H$ is an interpretation of $P=W_{1} \cup W_{2} \cup \ldots \cup W_{n}$. Therefore $P$ is a predecessor of $G$, which completes the proof.

Now the proof of the Main Theorem consists of constructing infinitely many weak predecessors for all graphs. We show this explicitely for $\mathrm{C}_{3}$.

Lemma 3 :

$\mathrm{C}_{3}$ has infinitely many weak predecessors.

Proof(outline): Construction of a set of graphs $\left\{S_{n}\right\}=3,5,7,9 \ldots$ which are weak predecessors of $\mathrm{C}_{3}$ :

(i) take a cycle $C_{3 n}$ ( $n$ an odd integer) and a vertex ' $n$ ' and insert edges between ' $n$ ' and every third vertex in $C_{3 n}$.

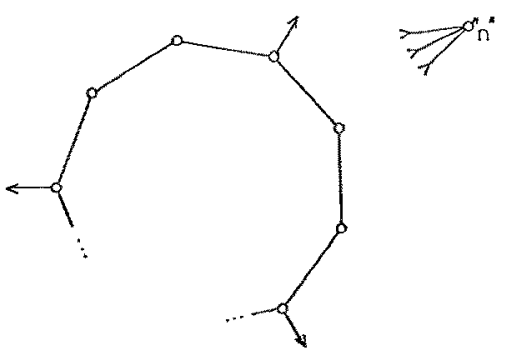


(ii) color the graph with the numbers 1,2, and 3 as follows:

- ' $n$ ' is 2-colored,

- a11 vertices which are adjacent to ' $n$ ' are 1 -colored,

- all vertices which follow a 1 -colored vertex in $\mathrm{C}_{3 \mathrm{n}}$ clockwise are 2-colored,

- the remaining vertices are 3-colored.

(iii) insert edges between every 3-colored and every 2-colored vertex (not equal to ' $n$ '), except those pairs which would form a $c_{3}$ with a 1 -colored vertex. This completes the construction of $S_{n}$.

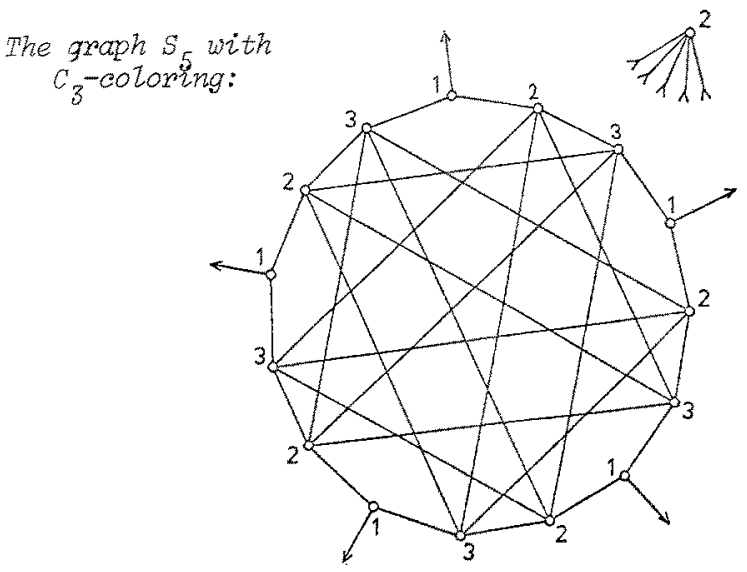

The identification of all vertices in $S_{n}$ which are colored with the same number results in a $C_{3}$. Thus $S_{n}$ is an interpretation of $C_{3} . C_{3}$ is not a subgraph of $S_{n}$ and every morphic image of $C_{3}$ contains a loop. Consequentiy, $C_{3}$ is not an interpretation of $S_{n}$ and we have the relation

$$
\mathcal{L}\left(s_{n}\right) c_{+} \mathcal{L}\left(c_{3}\right)
$$

Moreover, every elementary homomorphism on $S_{n}$ produces a morphic image $S_{n}$, which has a $C_{3}$ or a loop as a subgraph. Thus $\mathscr{L}\left(C_{3}\right)$ is a subset of $\mathcal{L}\left(S_{n}^{1}\right)$ and $\mathcal{L}\left(S_{n}^{1}\right) F_{f} \mathcal{L}\left(C_{3}\right)$ holds for no morphic image $S_{n}^{1}$ of $S_{n}$.

Similar constructions and Theorem 2 lead to

Lemma 4:

For $m \in N, C_{2 m+1}$ has no predecessor.

Consider an arbitrary graph $G$ with a minimal odd cycle of length $2 m+1$ (if a graph has no odd cycle, $G$ is bicolorable and $\mathcal{L}(G)=\mathcal{L}\left(K_{z}\right)$ ). We construct weak predecessors of $G$ by replacing an arbitrary $C_{2 m+1}$ by weak predecessors as described above. In this fashion the density of color-families can be shown. 


\section{Conclusion}

When we come back to the notion of grammar forms the result presented here can be shown to be equivalent to

$$
\text { "( } \left.\mathscr{L}\left(G_{1}\right), \mathscr{L}\left(G_{2}\right)\right) \text { is a dense pair" }
$$

where $G_{1}=(\{S\},\{a, b\},\{S+a b \mid b a\}, S)$ and

$G_{2}=(\{S\},\{a\},\{S+a a\}, S)$ are two finite context-free grammar forms. The existence of such a dense pair of finite grammar forms was an open problem in the theory of forms, stated e.g. in [3] and [5].

Using this approach, Maurer, Saloma and Wood, [4], have shown that $(\mathcal{L}(R E G), \mathcal{L}(C F))$ is not maximally dense.

Perhaps, some other open problems for grammar forms (like (i)

characterisation of subregular grammar forms, for which $(\mathcal{L}(G), \mathcal{L}(R E G)$ ) is dense, (ii) properties of minimal grammarforms which induce a grammatical family or ( $i j i)$ the complexity of membership in grammatical families) can be settled by a detour to "general coloring of graphs".

\section{References}

[1] Harary F., Graph Theory. Addison-Wesley (1972).

[2] Maurer H.A., Salomaa A., Wood D., Colorings and Interpretations: A Connection Between Graphs and Grammar Forms. Report 49, Institut für Informationsverarbeitung, TU Graz (1980).

[3] Maurer H.A., Salomaa A., Wood D., On Finite Gramar Forms. Report 51, Institut für Informationsverarbeitung, TU Graz (1980).

[4] Maurer H.A., Saloma A., Wood D., On Predecessors of Finite Languages. Report 57 , Institut für Informationsverarbeitung, TU Graz (1980).

[5] Salomaa A., Morphisms on Free Monoids and Language Theory, in: Formal Language Theory - Perspectives and open Problems, edited by R.V. Book. Academic Press (1980).

[6] Wood D., Grammar and L Forms: An Introduction. Springer Lecture Notes in Computer Science 91 (1980). 